\title{
QUASAR EMISSION LINE PROFILE MODELLING
}

T. R. Kallman

NASA/GSFC, Greenbelt, MD 20771

J. H. Krolik

Johns Hopkins University, Baltimore, MD 21218

B. J. Wilkes

Center for Astrophysics, 60 Garden St., Cambridge MA 02138

\section{Introduction}

We present fits of models for the C IV $\lambda 1549, \mathrm{~N} \mathrm{~V} \lambda 1240$ and $\mathrm{L} \alpha$ lines to observed profile data. Since these lines depend sensitively on the cloud ionization parameter (defined as the ratio of the illuminating flux to the cloud pressure; c.f. Krolik, McKee, and Tarter, 1981) and pressure, their relative strengths constrain the distribution of clouds in quasar broad line regions. We make the following assumptions: That the distribution of clouds around the continuum source is spherical and that the cloud motion relative to the continuum source is radial; that the clouds are in pressure equilibrium with hot intercloud mediurn (ICM) whose mass flux is conserved; and that the line fluxes emitted by the clouds as a consequence of the continuum illumination is given by "standard" (e.g. Kwan and Krolik, 1981) photoionization models. The cloud mass flux is not assumed to be constant, but is allowed to vary with velocity in order to fit the data. For the purposes of illustration, we have chosen a specific dynamical model: isothermal freefall with $\mathrm{T}_{I C M}=10^{8} \mathrm{~K}$. The cloud column density is assumed to be fixed at $10^{23} \mathrm{~cm}^{-2}$.

\section{Method}

The choice of a dynamical model determines the run of cloud velocity, $v(R)$,ionization parameter $\Xi(R)$, pressure $P(R)$, and size $D(R)$ with radius. Model line profiles are obtained by integrating the product of the number density of clouds, $N_{c \text { ! ouds }}(v(R))$, the cloud surface flux in line $\mathrm{j}$ (from photoionization models), $F^{(j)}(\Xi, P, D)$, and the cloud radiation pattern $f(\mu)$ over radius $\mathrm{R}$ and $\mu$ the cosine of the angle between the cloud velocity vector and the observer's line of sight

We vary $N_{\text {c'ouds }}(v(R))$ in order to give the best fit to observed profiles using a Maximum Entropy (ME) algorithm (Bryan and Skilling, 1980). We fit to observed profiles of C IV $\lambda 1549, L \alpha$ and $N$ V $\lambda 1240$ from the quasar PG1247+26. 


\section{Results}

The Figure displays the observed line profiles (crosses) along with the best fit models (solid line). Error estimates are derived from uncertainties in the continuum placement. The profiles of both $\mathrm{C}$ IV and $\mathrm{L} \alpha$ are approximately logarithmic, and both lines show blue asymmetry. We are able to obtain adequate agreement between models and data by varying the cloud distribution $N_{c \text { 'ords }}(v(R))$ and the cloud radiation pattern $f(\mu)$.

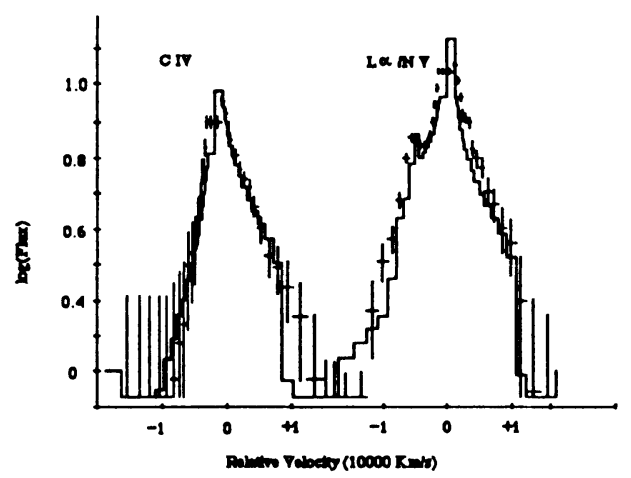

The ability of the models to fit the data is strongly affected by the line "peakiness", or the ratio of the intensity at line center to the width, and by the ratios of the strengths of the various lines. If the cloud emission were bolometric, i.e. Fine $\sim$ $\Xi P$ then $N_{\text {c:ouds }}(v(R)) \sim v^{3} \sim R^{-3 / 2}$ would produce logarithmic profiles similar to those observed. However, photoionization models produce $\mathrm{L} \alpha$ more efficiently at low ionization parameter, corresponding to low velocity ( $\Xi \sim R^{-1 / 2}$ for our choice of dynamics). Therefore a fit to the data requires that the $N_{c ! o x d s}(v)$ distribution be steeper than $v^{3}$. Furthermore, photoionization also produces greater L $\alpha / \mathrm{C} \mathrm{IV}$ and $L \alpha / \mathrm{N} V$ at low ionization parameter. The derived distribution is dominated by the highest velocity clouds, $N_{c ! o x d s}(v(R)) \sim v^{6-8}$.

The blue asymmetry implies non-isotropic cloud radiation pattern $f(\mu)$. C IV is adequately fit by clouds which radiate ispotropically from their illuminated side $f(\mu) \sim \min (1,1-\mu) . \quad \mathrm{L} \alpha$ is fit by clouds that radiate preferentially at an angle $30^{\circ}-45^{\circ}$ from their velocity vector. Standard clouds are expected to radiate approximately isotropically in C IV, and according to $f(\mu) \sim 1-\mu$ for $\mathrm{L} \alpha$. Anisotropic clouds must have opaque cores, and may also be elongated along their velocity vectors.

\section{References}

Bryan, R.K., and Skilling, J., 1980, M.N.R.A.S., 191, 69.

Krolik, J. H., McKee, C. F., and Tarter, C. B., 1981, Ap. J., 2ł9, 422.

Kwan, J., and Krolik, J. H., 1981, Ap. J., 250, 478. 